\title{
Uteroplacental Vessel Fibrinoid Necrosis with or without Atherosis
}

National Cancer Institute

\section{Source}

National Cancer Institute. Uteroplacental Vessel Fibrinoid Necrosis with or without

Atherosis. NCI Thesaurus. Code C118158.

The degeneration of an uteroplacental vessel with or without medial foamy macrophages. 